\title{
Synthetic pulsational line profile variations
}

\author{
R. Oreiro • J.H. Telting • R.H. Østensen $\cdot$ M. Briquet • \\ C. Aerts · U. Heber $\cdot$ M. Vučković
}

Received: 4 November 2009 / Accepted: 18 May 2010 / Published online: 28 July 2010

(C) Springer Science+Business Media B.V. 2010

\begin{abstract}
We have produced simulated time series of high resolution spectroscopy for sdBs. We present the first results of testing classical mode identification techniques. Here, in particular, we analyse the behaviour of individual spectral lines and discuss the results and applicability of the moment method.
\end{abstract}

Keywords Asteroseismology $\cdot$ Hot subdwarfs

\section{Introduction}

We understand by line profile variation (hereafter lpv) any change with time in spectral lines. This variation is due to a time dependent velocity field at the stellar surface. Moreover, spectral lines will also be affected by any effective temperature and $\log g$ variability.

If a star is undergoing stellar oscillations, its surface perturbations can be expressed in terms of spherical harmonics,

R. Oreiro $(\bowtie) \cdot$ R.H. Østensen · M. Briquet · C. Aerts ·

M. Vučković

Institute of Astronomy, Katholieke Universiteit Leuven,

Celestijnenlaan 200D BUS 2401, 3001 Leuven, Belgium

e-mail: raquel@ster.kuleuven.be

C. Aerts

Departement Astrofysica, IMAPP, Radboud Universiteit

Nijmegen, P.O. Box 9010, 6500 GL Nijmegen, The Netherlands

J.H. Telting

Nordic Optical Telescope, Aptdo. 474, 38710 Santa Cruz de la

Palma, Spain

U. Heber

Dr. Remeis-Sternwarte, Universität Erlangen-Nürnberg, Erlangen

Germany which are defined as a function of the quantum numbers $\ell$ and $m$. The oscillations can be visualised as a collection of surface areas moving in and out, which produces a complex velocity field, causing characteristic spectroscopic Doppler shifts. Depending on the geometry of the mode of oscillation, the line profile variations will be different. Ideally, then, observing lpv will help to identify $\ell$ and $m$ of the mode. This method requires, however, high signal to noise, high resolution spectroscopy, a challenge for the particular case of pulsating hot subdwarfs, which are faint $(B>12)$ and have short periods $(\sim 100-400 \mathrm{~s})$.

Pulsations in B-type hot subdwarfs (sdBs) were first discovered in 1997 (Kilkenny et al. 1997). Two subgroups of sdB pulsators are distinguished: short ( $p$-mode) and long ( $g$-mode) period pulsators (see Østensen 2009). The latter have lower pulsation amplitudes $(\sim 1 \mathrm{mmag})$ than the former. There exist, however, a few hybrid cases (Schuh et al. 2006; Lutz et al. 2008), since the two instability regions slightly overlap. Up to now, the deeper observational effort has focused on the short-period pulsating sdBs, with the organization of multi-site photometric campaigns (e.g. Kilkenny et al. 2003; Silvotti et al. 2006). With the detection of sufficient excited periods with high accuracy, the first seismic solutions have been possible (e.g. Brassard et al. 2001; Charpinet et al. 2006). They are based on the so called forward-method, in which all the observed periods are simultaneously matched to those computed for a grid of sdB structural models. No a priori knowledge on the mode geometry $(\ell, m)$ is assumed, although $T_{\text {eff- }} \log g$ are commonly used to constrain the solution. However, the importance of observational mode identification in the seismic analysis has been theoretically (Hu et al. 2008) and observationally (e.g. Randall et al. 2009) confirmed.

A recent observational effort is being made to detect and use lpv for an empirical mode identification in sdBs 
(Vučković et al. 2009; Telting et al. 2008). In this work we present the first results on using simulated $1 \mathrm{pv}$ as input for classical mode identification techniques, in particular the moment method (Briquet and Aerts 2003). Some related work has been presented by Schoenaers and LynasGray (2008).

\section{Observational data}

Within this framework, the target Balloon 090100001 seems to be the most suitable pulsating sdB for studying line profile variations. It is the brightest pulsating $\operatorname{sdB}(B=11.8)$, showing both $p$-and $g$-mode pulsations (Oreiro et al. 2005). Among $p$-mode sdB pulsators, this target has one of the longest periods of oscillation ( $356 \mathrm{~s})$, allowing relatively long exposure times to be taken, helping to increase the signal to noise. Moreover, the star has a high amplitude of oscillation for the dominant mode ( $\sim 50 \mathrm{mma})$.

With the intention of detecting line profile variations in this target, and attempt a mode identification, 6 nights of time series high resolution spectroscopy were acquired in 2006 at the $2.6 \mathrm{~m}$ Nordic Optical Telescope. In total, 1606 spectra were obtained with $30 \mathrm{~s}$ exposure time. Table 1 includes the log of the observations. Note that the first night was in August, while the last one in December the same year.

The first two nights were already analysed by Telting et al. (2008). They considered the main oscillation period, and combined all the frames at similar oscillation phase, in order to increase the $\mathrm{S} / \mathrm{N}$. Moreover, all the metal lines present in the spectra were combined through cross-correlation to further improve the $\mathrm{S} / \mathrm{N}$, producing 12 cross-correlation profiles. They concluded the mode must be an $\ell=0$ or $\ell=1$ mode.

Here we proceed with a different approach. We have worked with all the 6 nights, producing 1606 low S/N spectra. Figure 1 shows some wavelength portions of the mean spectrum, where some metal lines are distinguished. After identifying the spectral lines present, they were combined through cross-correlation to produce 1606 cross-correlation profiles of still low signal to noise, but of sufficient quality to perform a frequency analysis.

A Fourier analysis along the profile (for each velocity bin) was computed using the software package FAMIAS (Zima 2008), showing that oscillation power is present in a velocity range $(-50,50) \mathrm{km} / \mathrm{s}$. The clearest feature in the Fourier domain is the main dominant mode of the star $\left(f_{1}\right)$, although the frequency analysis revealed five modes of oscillations, two more peaks at the $p$-mode zone, and two being at the $g$-mode frequency region. They are listed in Table 2, including two additional combination frequencies.

Once the frequencies have been accurately determined (note the large time coverage of the data), we have used $f_{1}$ to
Table 1 Log of observations: number of spectra acquired each night

\begin{tabular}{lc}
\hline Date (2006) & \#Frames \\
\hline August, 3 & 385 \\
August, 4 & 457 \\
October, 23 & 255 \\
November, 9 & 170 \\
December, 25 & 84 \\
December, 26 & 255 \\
\hline
\end{tabular}

Table 2 List of frequencies detected from the whole 1606 cross-correlation profiles

\begin{tabular}{ll}
\hline Freq. $(\mu \mathrm{Hz})$ & \\
\hline 2807.44 & $f_{1}$ \\
2820.65 & $f_{2}$ \\
2824.88 & $f_{3}$ \\
325.61 & $f_{4}$ \\
363.78 & $f_{5}$ \\
5614.89 & $2 \cdot f_{1}$ \\
5632.32 & $f_{1}+f_{2}$ \\
\hline
\end{tabular}
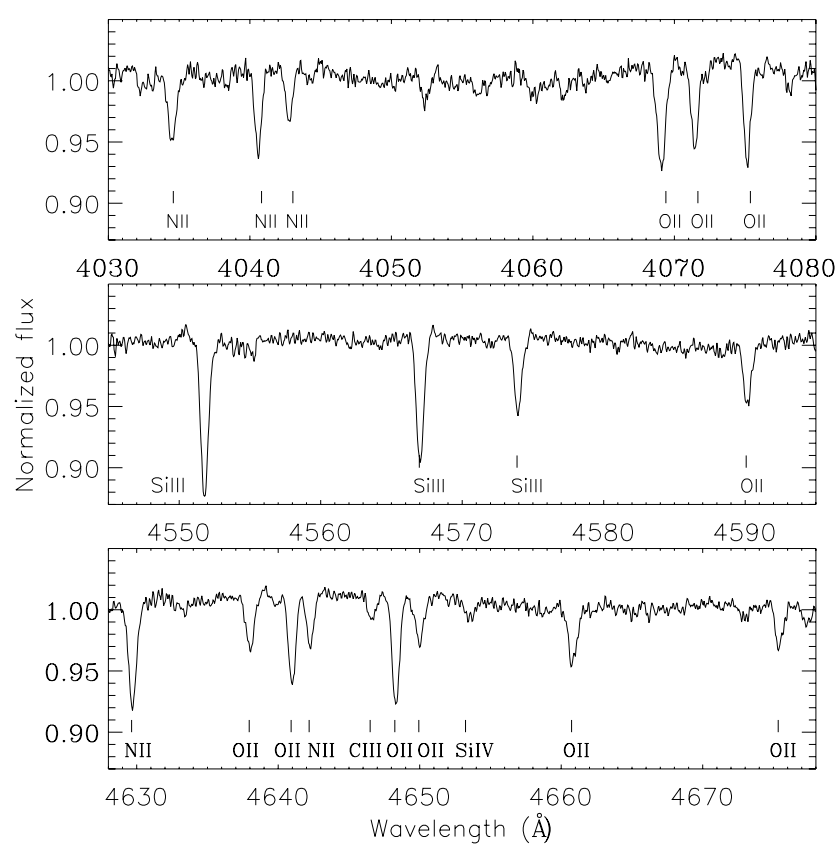

Fig. 1 Mean spectrum of the complete data set in Table 1. Some metal lines are indicated

phase-fold the individual spectra into 24 spectra equally distributed along the $f_{1}$ pulsation phase. Furthermore, a crosscorrelation profile was generated for each of the phasefolded spectrum, combining the information on all metallic lines into a single, artificial profile, and thus increasing the signal to noise.

Based on these 24 cross-correlation profiles, we have next used the discriminant code of Briquet and Aerts (2003) 
to obtain the most probable mode identification for the dominant frequency observed in Balloon 090100001. It makes use of the different response of a spectral line, described as its first three moments, to different modes of oscillation

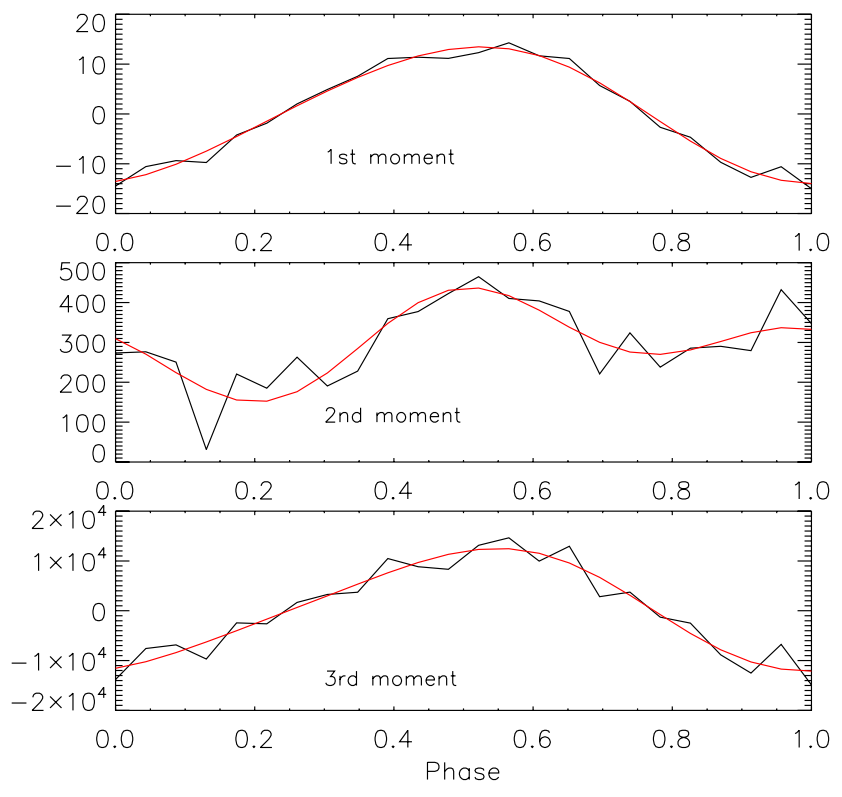

Fig. 2 First three moments computed for the cross-correlation profiles obtained from the 24 spectra folded to the phase of $f_{1}$ $(\ell, m)$. The first three moments computed for $f_{1}$ are displayed in Fig. 2.

$f_{1}$ has already been identified as a radial mode $(\ell=0)$ using different techniques (Baran et al. 2008; Charpinet et al. 2008). Using the moment method (Briquet and Aerts 2003), the results we obtain can only constrain the mode to have $\ell<3,|m|<2$, since we get similar discriminant values for all these possible mode identification, whilst a much higher discriminant value (less probable identification) for higher $\ell$ and $m$ values. An insufficient signal to noise, an information degradation in the phase-folding and/or crosscorrelation processes, or some physical effects not considered, may be hampering a clearer mode identification.

\section{Synthetic line profile variations}

The poor constraint obtained for $\ell$ and $m$ motivated the simulations described in this section, where we present the preliminary results. This work aims to check the feasibility of the mode identification method used in the previous section with the observations.

A suitable model atmosphere grid was used as input for the codes Bruce and Kylie (Townsend 1997), which produced a synthetic time series of high resolution spectra. A radial mode was imposed in order to check whether a
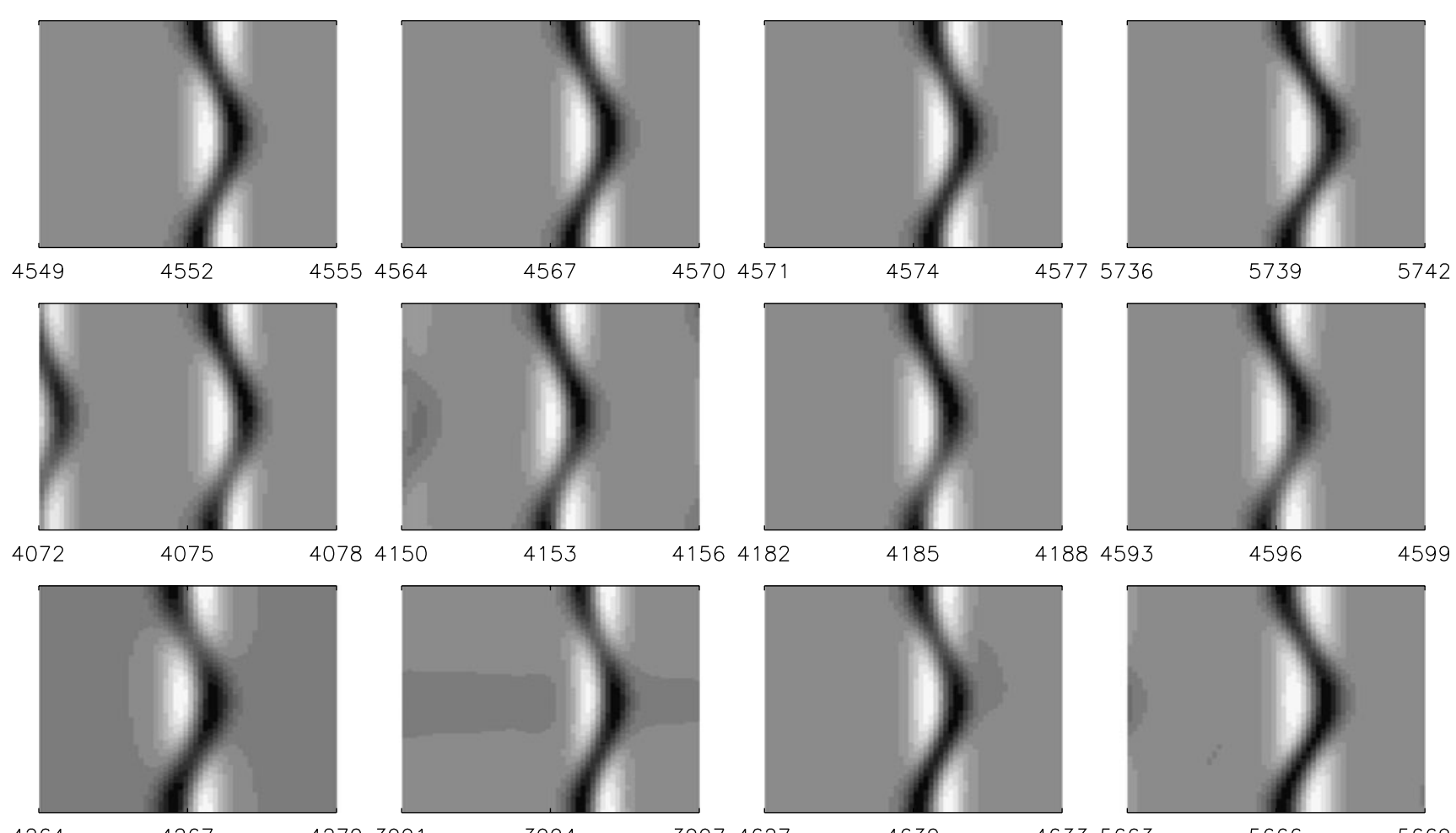

41564182

4185

41884593

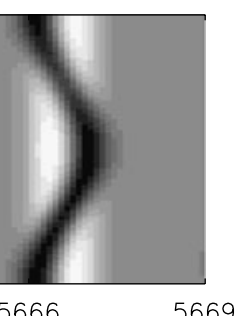

Fig. 3 Greyscale plots for some spectral lines from the synthetic time series spectroscopy. SiIII (top row), OII (second row), and CII and NII lines (bottom row) 

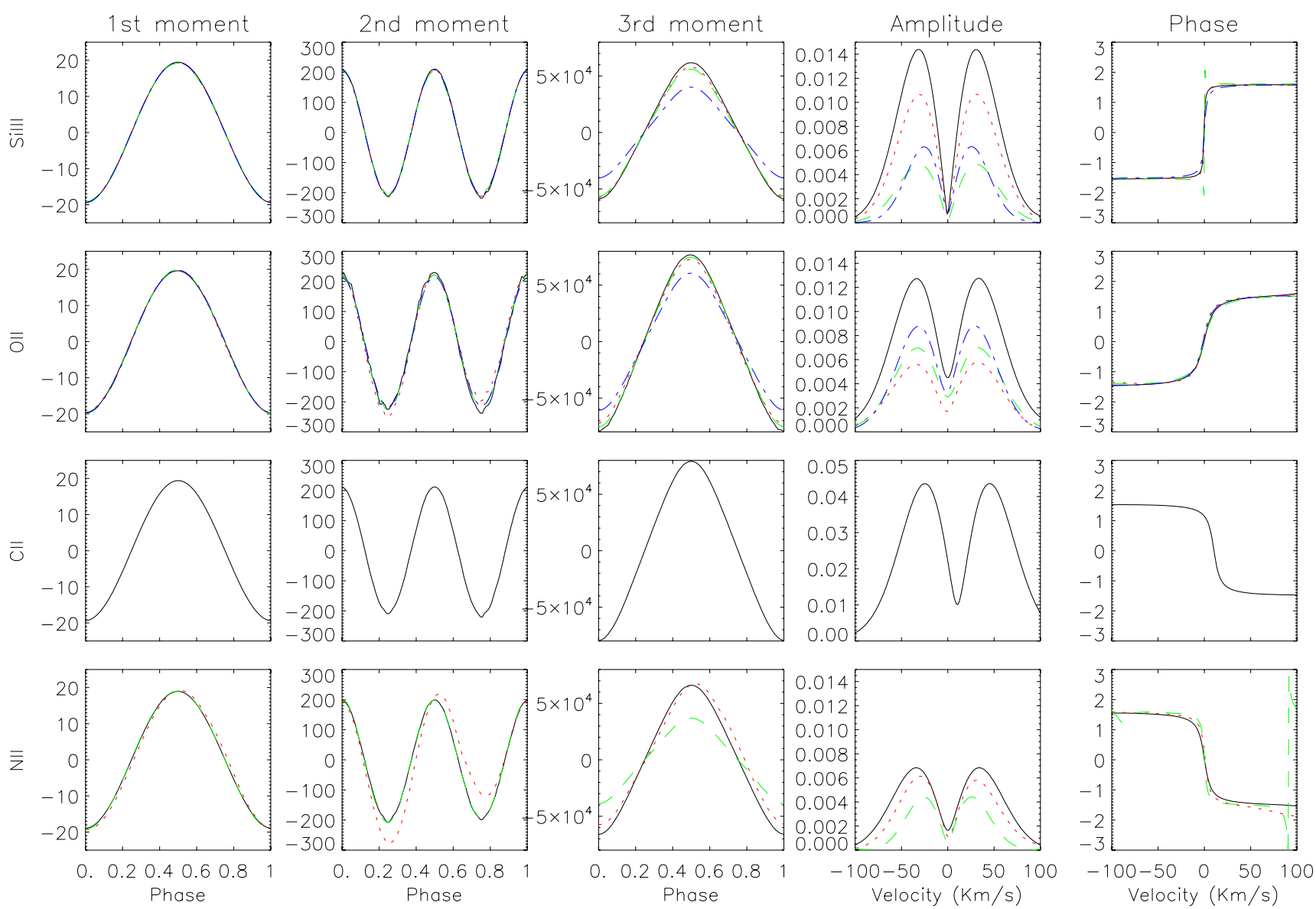

Fig. 4 From left to right: first, second and third moments, and amplitude and phase across the profile, for spectral lines of different ions (from top to bottom). Different colors in the same panel represent different lines of the same ion

correct mode identification is retrieved using the moment method.

We have first examined the lpv for some individual spectral lines. Figure 3 displays the greyscale plots for some SiIII (top row), OII (second row), and CII and NII lines (bottom row). They are produced after subtracting the mean profile to the time series, so that the variations can be easily seen. Note that not all lines behave exactly the same, mainly due to the different response of their equivalent widths to temperature variations during the oscillation phase.

For each line under consideration, the first three moments and the amplitude/phase across the profile were computed (Fig. 4), although in this work we only make use of the moments, as input for the mode identification discriminant of Briquet and Aerts (2003).

The results of the mode identification are summarized in Fig. 5. It shows the discriminant value for each possible $(\ell, m)$; the lower the discriminant, the higher the probability for this identification. The discriminant for lines of the same ion are included in a same panel. Since a radial mode was imposed, we expect a minimum at $\ell=0$, indicated by a vertical dash-dotted line. We can see that this is in gen- eral the case, although some lines behave better in the sense they achieve a clearer minimum at the right mode identification. Regarding their similar discriminant values, however, it is difficult to assess whether a radial mode is preferred to $\ell=1,2$ identifications, which seems to be in agreement with the observational results.

\section{Conclusions}

We present the first results on the application of the moment method for pulsating B-type hot subdwarfs. We have produced synthetic time-series high resolution spectroscopy, imposing a radial mode of oscillation, in order to check if the mode identification technique retrieves the right identification. As a first approach, we have focused on individual spectral lines. Their variations were correctly identified as due to a radial mode, although $\ell=1$ and some $\ell=2$ modes can not be discarded for most of the spectral lines.

As expected, we note the method is highly sensitive to the normalization procedure, which is particularly delicate for shallow lines and/or when nearby lines exist. We plan 

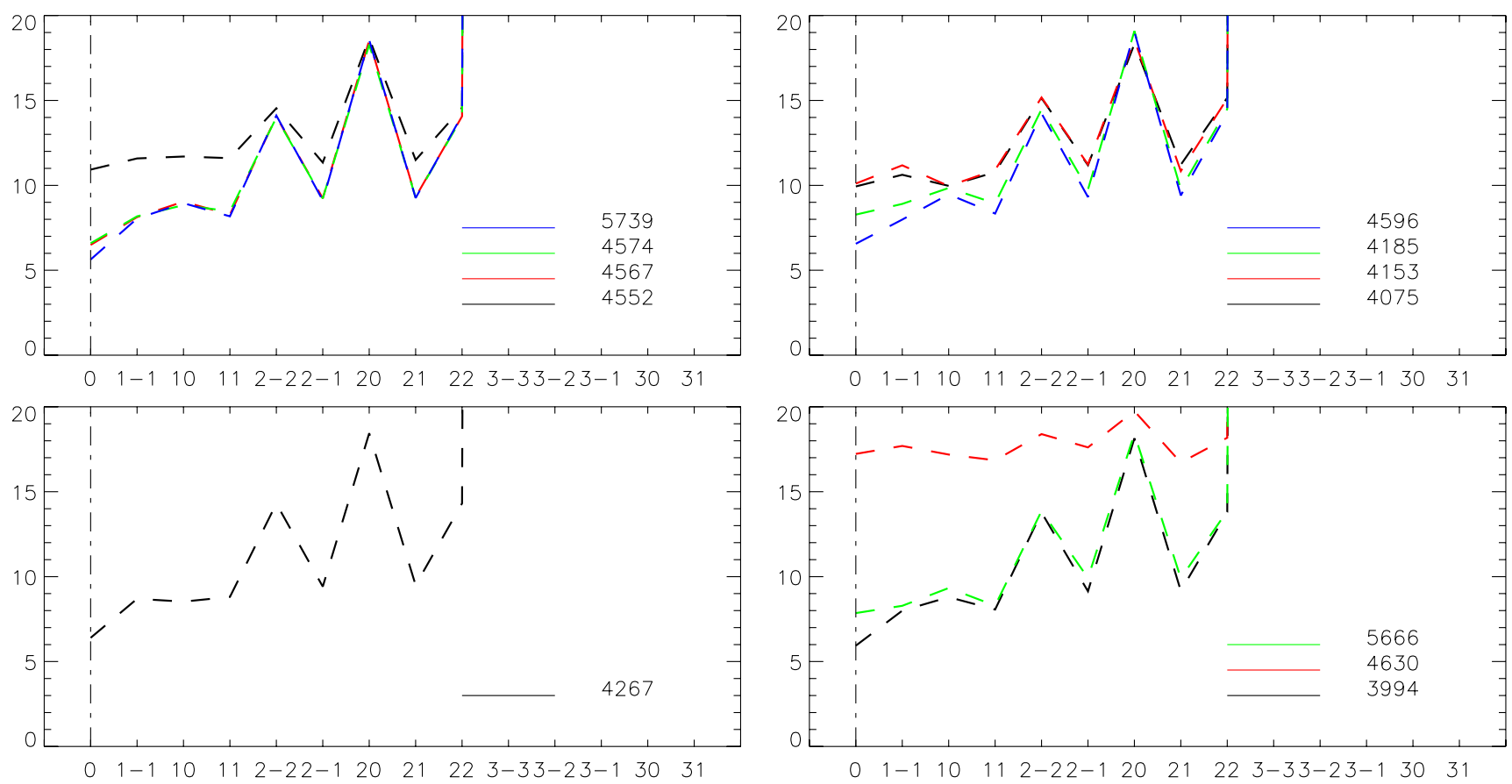

Fig. 5 Discriminant value as function of possible mode identifications, obtained using the moment method. Each panel includes the results for spectral lines of the same ion, from up to down and left to

to increase the number of spectral lines under study, paying attention to their specific sensitivity to temperature effects. The next step is to check how the information is affected when different lines are merged through cross-correlation, and what is the influence on mode identification, if any. This may shed light on the observational requirements for a correct use of the mode identification technique for the case of hot subdwarfs.

Acknowledgements The research leading to these results has received funding from the European Research Council under the European Community's Seventh Framework Programme (FP7/20072013)/ERC grant agreement $n^{\circ} 227224$ (PROSPERITY), as well as from the Research Council of K.U.Leuven grant agreement GOA/ 2008/04. MB is Postdoctoral Fellow of the Fund for Scientific Research, Flanders.

\section{References}

Baran, A., Pigulski, A., O’Toole, S.J.: Mon. Not. R. Astron. Soc. 385, 255 (2008). doi:10.1111/j.1365-2966.2008.12662.x

Brassard, P., Fontaine, G., Billères, M., Charpinet, S., Liebert, J., Saffer, R.A.: Astrophys. J. 563, 1013 (2001). doi:10.1086/323959

Briquet, M., Aerts, C.: Astron. Astrophys. 398, 687 (2003). doi:10. 1051/0004-6361:20021683

Charpinet, S., Silvotti, R., Bonanno, A., Fontaine, G., Brassard, P., Chayer, P., Green, E.M., Bergeron, P., Bernabei, S., Leccia, S., Kjeldsen, H., Janulis, R., Frasca, A., Østensen, R., Kim, S., Park, B., Jiang, X., Reed, M.D., Patterson, R.S., Gietzen, K.M., Clark,

right: SiIII, OII, FeIII, CII and NII lines. The correct mode identification $(\ell=0)$ is indicated with a dash-dotted vertical line. The lower the discriminant is, the higher the probability for this mode identification

P.J., Wolf, G.W., Lipkin, Y., Formiggini, L., Leibowitz, E., Oswalt, T.D., Rudkin, M., Johnston, K.: Astron. Astrophys. 459, 565 (2006). doi:10.1051/0004-6361:20065316

Charpinet, S., Fontaine, G., Brassard, P., Randall, S.K., van Grootel, V., Green, E.M., Chayer, P.: In: U. Heber, C. S. Jeffery, \& R. Napiwotzki (ed.) Hot Subdwarf Stars and Related Objects. Astronomical Society of the Pacific Conference Series, vol. 392, p. 297 (2008)

Hu, H., Dupret, M., Aerts, C., Nelemans, G., Kawaler, S.D., Miglio, A., Montalban, J., Scuflaire, R.: Astron. Astrophys. 490, 243 (2008). doi:10.1051/0004-6361:200810233

Kilkenny, D., Koen, C., O’Donoghue, D., Stobie, R.S.: Mon. Not. R. Astron. Soc. 285, 640 (1997)

Kilkenny, D., Reed, M.D., O’Donoghue, D., Kawaler, S.D., Mukadam, A., Kleinman, S.J., Nitta, A., Metcalfe, T.S., Provencal, J.L., Watson, T.K., Sullivan, D.J., Sullivan, T., Shobbrook, R., Jiang, X.J., Joshi, S., Ashoka, B.N., Seetha, S., Leibowitz, E., Ibbetson, P., Mendelson, H., Meištas, E., Kalytis, R., Ališauskas, D., Martinez, P., van Wyk, F., Stobie, R.S., Marang, F., Zola, S., Krzesinski, J., Ogłoza, W., Moskalik, P., Silvotti, R., Piccioni, A., Vauclair, G., Dolez, N., Chevreton, M., Dreizler, S., Schuh, S.L., Deetjen, J.L., Solheim, J., Gonzalez Perez, J.M., Ulla, A., Østensen, R., Manteiga, M., Suarez, O., Burleigh, M., Kepler, S.O., Kanaan, A., Giovannini, O.: Mon. Not. R. Astron. Soc. 345, 834 (2003). doi:10. 1046/j.1365-8711.2003.07007.x

Lutz, R., Schuh, S., Silvotti, R., Dreizler, S., Green, E.M., Fontaine, G., Stahn, T., Hügelmeyer, S.D., Husser, T.: In: U. Heber, C. S. Jeffery, \& R. Napiwotzki (eds.) Hot Subdwarf Stars and Related Objects. Astronomical Society of the Pacific Conference Series, vol. 392 , p. 339 (2008)

Oreiro, R., Pérez Hernández, F., Ulla, A., Garrido, R., Østensen, R., MacDonald, J.: Astron. Astrophys. 438, 257 (2005). doi:10.1051/ 0004-6361:20052681

Østensen, R.H.: Commun. Asteroseismol. 159, 75 (2009) 
Randall, S.K., van Grootel, V., Fontaine, G., Charpinet, S., Brassard, P.: Astron. Astrophys. 508, 911-921 (2009). doi:10.1051/ 0004-6361/200912576

Schoenaers, C., Lynas-Gray, A.E.: In: U. Heber, C. S. Jeffery, \& R. Napiwotzki (eds.) Hot Subdwarf Stars and Related Objects. Astronomical Society of the Pacific Conference Series, vol. 392, p. 253 (2008)

Schuh, S., Huber, J., Dreizler, S., Heber, U., O’Toole, S.J., Green, E.M., Fontaine, G.: Astron. Astrophys. 445, 31 (2006). doi:10. 1051/0004-6361:200500210

Silvotti, R., Bonanno, A., Bernabei, S., Fontaine, G., Charpinet, S., Leccia, S., Kjeldsen, H., Janulis, R., Frasca, A., Østensen, R., Kim, S., Park, B., Jiang, X., Reed, M.D., Patterson, R.S., Giet- zen, K.M., Clark, P.J., Wolf, G.W., Lipkin, Y., Formiggini, L., Leibowitz, E., Oswalt, T.D., Rudkin, M., Johnston, K., Brassard, P., Chayer, P., Green, E.M., Bergeron, P.: Astron. Astrophys. 459, 557 (2006). doi:10.1051/0004-6361:20065314

Telting, J.H., Geier, S., Østensen, R.H., Heber, U., Glowienka, L., Nielsen, T., Oreiro, R., Frandsen, S.: Astron. Astrophys. 492, 815 (2008). doi:10.1051/0004-6361:200810759

Townsend, R.H.D.: Mon. Not. R. Astron. Soc. 284, 839 (1997)

Vučković, M., Østensen, R.H., Aerts, C., Telting, J.H., Heber, U., Oreiro, R.: Astron. Astrophys. 505, 239 (2009). doi:10.1051/0004 $-6361 / 200912457$

Zima, W.: Commun. Asteroseismol. 155, 17 (2008) 